\title{
Décisions concernant l'exposition chronique du public aux rayonnements : nouvelles recommandations de la CIPR
}

\author{
ABEL J. GONZÁLEZ*
}

(Manuscrit reçu le 31 octobre 2000)

\begin{abstract}
RÉSUMÉ On présente ici des recommandations que vient d'établir la Commission internationale de protection radiologique, pour un domaine qu'il est relativement difficile de délimiter, celui de l'exposition «prolongée » (il faut entendre par là « chronique ») du public aux rayonnements ionisants, dans le cas de niveaux de dose relativement bas. Le rapport de la Commission est fondé sur les travaux d'un groupe de travail de six personnes, présidé par l'auteur. Ce rapport doit être considéré comme contenant des recommandations d'aide à la décision, les résultats devant, en principe, servir au processus final de prise de décision, plus large.
\end{abstract}

\section{Introduction}

Cette communication résume les recommandations qui figurent dans un nouveau rapport publié par la Commission internationale de protection radiologique (CIPR, 1999). Ce rapport pourrait avoir des conséquences d'une grande portée pour les décideurs qui s'occupent d'un sujet difficile à cerner : l'exposition chronique et persistante (ou ce que le rapport appelle l'exposition prolongée) du public à des niveaux relativement bas de rayonnements ionisants (en bref, rayonnements). Le rapport, intitule «Protection of the public in situations of prolonged radiation exposure: the application of the commission's system of radiological protection to controllable radiation exposure due to natural sources and long lived radioactive residues » (Protection du public dans des situations d'exposition prolongée à des rayonnements : application du système de protection radiologique de la Commission aux expositions maîtrisables dues à des sources naturelles et à des résidus radioactifs à longue période), a été approuvé par la Commission à sa réunion, tenue à Saint-Pétersbourg (Fédération de Russie) en septembre 1999. Le rapport a été élaboré par un groupe de travail ad hoc créé par la CIPR sur l'avis du Comité 4 de la Commission, et il a été approuvé par le Comité à sa réunion de Kiawah (États-Unis), en mars 1999. Les membres du groupe étaient : John Cooper, Per Hedemann-Jensen, Sieglinde Przyborowski, Michael Savkin, John Till et

* Agence internationale de l'énergie atomique, Wagramer Strasse 5, BP 100, 1400 Vienne, Autriche. 
l'auteur de la présente communication, qui a eu l'honneur de présider les travaux du groupe. Cette communication est fondée principalement sur la synthèse du rapport.

\section{Exposition prolongée}

Les personnes du public sont soumises fortuitement et durablement à des expositions prolongées qui sont liées à des situations dans lesquelles elles peuvent se trouver placées. La dose annuelle moyenne associée à des expositions prolongées est relativement constante ou décroît progressivement au fil des ans. (Le rapport ne couvre pas les expositions passagères du public, ni les expositions professionnelles et médicales, qui ne sont pas considérées comme des expositions prolongées.) Les expositions prolongées sont en général celles qui sont dues aux sources dites «naturelles » comme le rayonnement cosmique et les chaînes de désintégration des radionucléides naturels. Certaines sources «artificielles » peuvent aussi donner lieu à des expositions prolongées. Par exemple, des déchets radioactifs de longue période résultant d'activités humaines sont une cause fréquente d'exposition prolongée. Il convient de noter que les déchets radioactifs peuvent contenir des radionucléides naturels et artificiels. Dans des situations d'exposition prolongée, il peut être difficile de distinguer les expositions imputables à la composante artificielle de celles dues à la composante naturelle ; en outre, les mesures de protection contre la composante artificielle peuvent influer sur l'exposition due à la composante naturelle, et vice versa.

\section{Pratiques et interventions}

Le système de protection radiologique se fonde sur les concepts de «pratiques » et d' "interventions », qui s'appliquent pleinement aux situations d'exposition prolongée. Des déchets radioactifs peuvent être encore présents après la fin d'actions réglementées qui augmentent l'exposition globale de la population aux rayonnements. Ces actions sont appelées pratiques. Les déchets peuvent aussi provenir d'actions et d'événements non réglementés. L'exposition à des sources naturelles et à des déchets radioactifs qui existent déjà - de facto - dans l'habitat humain peut aussi faire l'objet d'actions protectrices grâce à un processus dit d'intervention destiné à diminuer l'exposition globale de la population.

\section{Exposition prolongée maîtrisable}

De nombreuses expositions prolongées à des sources naturelles et presque toutes les expositions prolongées à des déchets radioactifs sont maîtrisables. Les expositions prolongées qui sont essentiellement non maîtrisables (par exemple 
l'exposition à des radionucléides naturels qui modifient le métabolisme humain) ou qu'il n'est pas raisonnable de maîtriser (par exemple l'exposition au rayonnement cosmique) sont généralement exclues du champ de réglementation concernant la protection radiologique. Les sources d'exposition prolongée maîtrisable comprennent donc certains radionucléides qui se trouvent dans la nature et pour lesquels il est possible de maîtriser l'exposition, et les déchets radioactifs de périodes longues. Des déchets radioactifs peuvent être encore présents dans l'habitat humain après l'exécution et le déclassement de pratiques réglementées; il en est de même pour d'autres activités menées hors de tout contrôle ou dans le cadre de prescriptions réglementaires moins rigoureuses que celles que l'on applique aujourd'hui. Ils peuvent aussi résulter d'événements d'accidents par exemple - qui entraînent des rejets de radionucléides dans l'environnement. Les produits de consommation courante contenant des substances radioactives peuvent aussi être à l'origine d'expositions prolongées.

\section{Grandeurs}

La grandeur recommandée dans le rapport pour évaluer les situations d'exposition prolongée est la dose annuelle [efficace] imputable à l'exposition. Une grandeur subsidiaire est la somme des doses annuelles causées par toutes les sources d'exposition prolongée qui persistent dans un habitat humain donné; cette grandeur est appelée la dose annuelle existante. La dose annuelle qui est ajoutée à la dose annuelle existante imputable à une pratique s'appelle la dose annuelle supplémentaire. La dose annuelle qui est soustraite à la dose annuelle existante du fait d'une intervention est appelée la dose annuelle évitée. (Si l'on veut parler d'une dose annuelle qui pourra être empêchée grâce à une intervention à venir, on emploie l'expression dose annuelle évitable.)

\section{Application aux pratiques}

Les principes du système de protection radiologique s'appliquant aux pratiques sont la justification de la pratique, l'optimisation de la protection radiologique, s'agissant des sources liées aux pratiques, et la limitation des doses individuelles imputables à la pratique. Ces principes devraient être appliqués, à titre prévisionnel, dès le stade de la planification de toute pratique - notamment de celles qui sont susceptibles de donner lieu à des expositions prolongées - à la conception, à l'exécution et au déclassement des pratiques et des sources de rayonnement qui leur sont associées. Avant l'introduction d'une pratique entraînant une exposition prolongée, il y a une dose annuelle existante; après le déclassement, il reste une dose annuelle prolongée supplémentaire imputable à cette pratique (Fig. 1). Les principes s'appliquent au contrôle de cette dose 


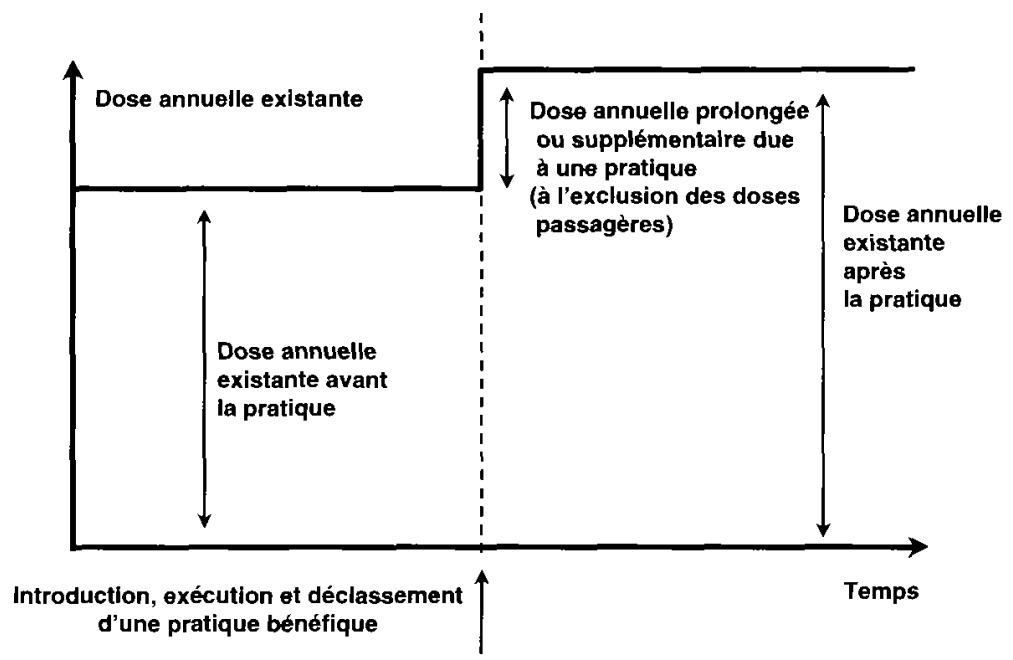

Figure 1 - Présentation schématique de la dose annuelle existante avant et après une pratique.

annuelle supplémentaire et pas à la dose annuelle existante. Dans certaines conditions, des sources utilisées dans le cadre de pratiques justifiées peuvent être exemptées des prescriptions réglementaires si les doses annuelles supplémentaires individuelles attribuables à la source sont inférieures à $0,01 \mathrm{mSv}$ par an environ.

\subsection{Justification des pratiques}

La justification d'une pratique entraînant une exposition prolongée suppose que tous les facteurs à long terme qui entrent en jeu soient analysés avant que la pratique ne soit adoptée. Les facteurs pertinents sont ceux qui concernent les substances radioactives de période longue susceptibles d'être rejetées dans l'environnement ou de subsister dans l'habitat humain comme déchets radioactifs après le déclassement de la pratique. Ils comprennent les composantes prolongées des doses annuelles supplémentaires escomptées, tant individuelles que collectives, qui sont attribuables aux rejets et aux déchets.

\subsection{Optimisation de la protection}

L'optimisation de la protection suppose que l'on choisisse la meilleure option de protection radiologique pour une source, eu égard à la situation sociale et économique du moment. Cette option optimale devra maintenir les doses «au niveau le plus bas qu'il est raisonnablement possible d'atteindre» (principe ALARA), compte tenu des facteurs économiques et sociaux. Dans le cas d'une 
pratique justifiée entraînant une exposition prolongée, tous les facteurs à long terme pertinents devraient être pris en considération dans le processus d'optimisation. Ce processus peut être appliqué en suivant les techniques d'optimisation recommandées par la Commission.

\subsection{Limitation de la dose individuelle}

L'application des principes de justification et d'optimisation aux pratiques peut donner lieu à des inégalités entre les individus qui peuvent être importantes s'agissant d'expositions prolongées. Ces inégalités sont dues au fait que les expositions peuvent concerner une vaste zone et toucher des gens qui ne bénéficient pas directement de la pratique. Elles peuvent aussi résulter du fait que les expositions prolongées peuvent être de longue durée, ce qui peut affecter les générations futures. (Le rapport note cependant que les inégalités entre les générations sont un concept plus flou que les inégalités entre les individus à un moment donné.) Pour limiter ces inégalités et tenir compte des expositions prolongées et passagères à des sources multiples, le rapport recommande d'appliquer rigoureusement les restrictions de doses individuelles aux expositions prolongées qui devraient provenir des différentes sources ainsi qu'à la somme des expositions prolongées qui devraient résulter de l'ensemble des pratiques réglementées. Les restrictions concernant l'exposition aux sources s'appellent contraintes de dose; les restrictions concernant l'exposition due aux pratiques s'appellent limites de dose.

\subsubsection{Contraintes de dose}

En ce qui concerne les contraintes de dose, le rapport confirme la recommandation de la CIPR selon laquelle la valeur maximale de la contrainte de dose à utiliser dans l'optimisation de la protection radiologique pour une source unique devrait être inférieure à $1 \mathrm{mSv}$ par an, une valeur ne dépassant pas $0,3 \mathrm{mSv}$ environ en une année étant appropriée. Il insiste par ailleurs sur la nécessité de prendre en considération les situations dans lesquelles des expositions passagères et prolongées peuvent se combiner, ou celles où des expositions prolongées dues à une seule source peuvent s'accumuler à la longue. Dans de tels cas, le rapport recommande de vérifier que des méthodes appropriées soient employées pour évaluer la dose afin de s'assurer de la conformité à la contrainte de dose établie. L'évaluation devrait prendre en compte toutes les combinaisons et tous les cumuls d'exposition que l'on peut raisonnablement envisager. $\mathrm{Si}$, dans une situation donnée, on ne peut pas vérifier la conformité à la contrainte de dose, le rapport considère qu'il est prudent de réduire la composante prolongée de la dose individuelle reçue d'une source en appliquant une contrainte de dose de l'ordre de 
0,1 mSv durant n'importe quelle année, pendant la durée de vie opérationnelle de la source.

\subsubsection{Limites de dose}

Pour ce qui est des limites de dose, le rapport confirme également la recommandation de la CIPR tendant à ce que la somme des expositions prolongées et passagères dues à l'ensemble des pratiques réglementées ne dépasse pas $1 \mathrm{mSv}$ par an. Il souligne également que les autorités nationales concernées et, le cas échéant, les organisations internationales intéressées devraient prendre en considération les situations dans lesquelles les composantes prolongées des expositions dues à l'ensemble des pratiques réglementées pourraient s'ajouter les unes aux autres par suite d'une accumulation des déchets radioactifs provenant d'opérations pratiques continues, l'objectif étant d'empêcher que les doses annuelles supplémentaires individuelles totalisées imputables à toutes les pratiques du moment et aux pratiques futures prévisibles ne dépassent la dose limite de $1 \mathrm{mSv}$ par an.

La figure 2 présente les recommandations chiffrées concernant les contraintes et les limites de doses pour les pratiques.

\section{Application aux interventions}

Les principes du système de protection radiologique s'appliquant aux interventions sont la justification des interventions et l'optimisation des actions protectrices. Ces principes devraient s'appliquer à toute situation de fait liée à une exposition prolongée maîtrisable lorsqu'une dose annuelle existante peut être en partie évitée grâce à une intervention (Fig. 3).

\subsection{Justification de l'intervention}

La justification d'une intervention dans des situations d'exposition prolongée devrait être évaluée grâce à un processus d'aide à la décision visant à ce qu'il y ait un bon équilibre entre toutes les dimensions à long terme de la protection radiologique. (Outre les doses annuelles évitables, individuelles et collectives, on peut citer, parmi les autres dimensions : la perspective de réduire l'inquiétude causée par la situation, le fait que l'intervention rassurera les personnes concernées diminuant ainsi le coût social, le détriment et les perturbations que peut entraîner la mise en œuvre d'actions protectrices.) Les résultats de ce processus d'aide à la décision devraient contribuer au processus décisionnel dans lequel d'autres éléments peuvent entrer en ligne de compte et auquel les parties concernées peuvent prendre part (voir perspectives, ci-après). 


\section{Restrictions de dose pour les pratiques : dose annuelle supplémentaire}

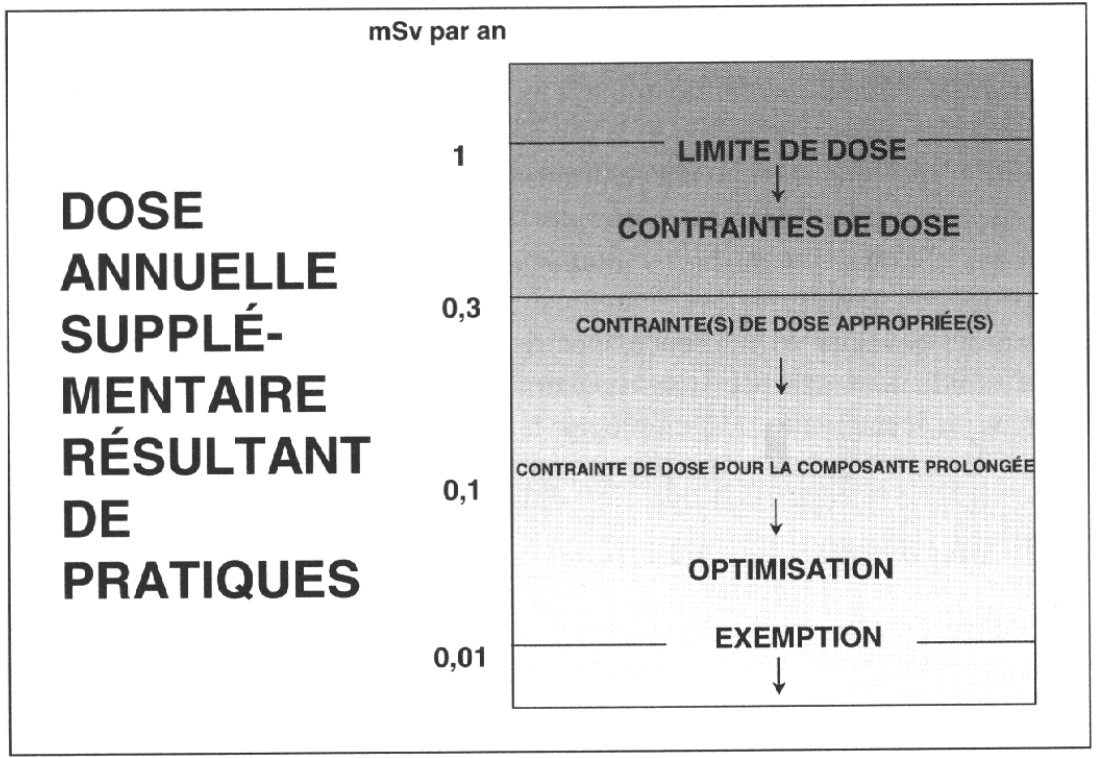

Figure 2-Recommandations chiffrées concernant les contraintes et les limites de dose pour les pratiques.

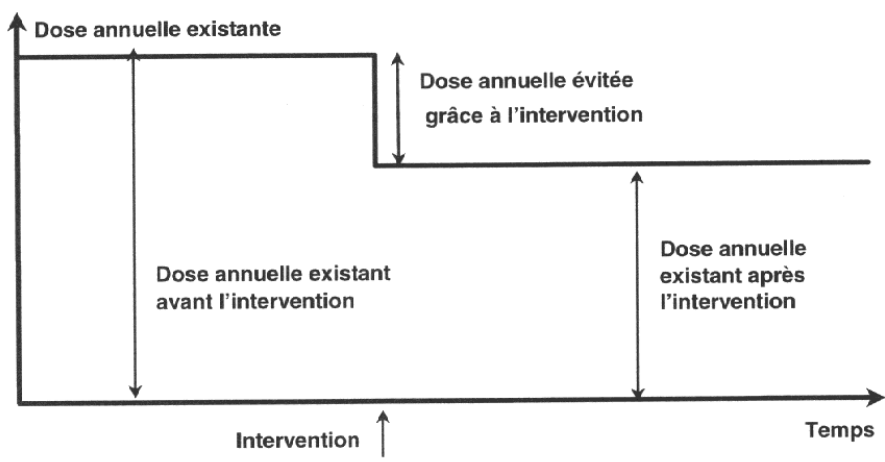

Figure 3 - Présentation schématique de la dose annuelle existant avant et après une intervention.

\subsection{Optimisation des actions protectrices}

On peut optimiser les actions protectrices en suivant la méthode générale d'optimisation concernant les pratiques recommandée par la CIPR. Leur forme, leur étendue et leur durée optimales devraient être choisies parmi les options 
d'intervention justifiées. Pour certaines situations d'exposition prolongée, le processus d'optimisation peut conduire à restreindre l'accès à l'habitat humain.

\subsection{Niveaux de référence spécifiques}

Les autorités nationales (et éventuellement les organisations internationales concernées) devraient déterminer à l'avance, pour des situations particulières d'exposition prolongée pouvant entraîner une intervention, des niveaux de référence spécifiques (comme des niveaux d'intervention, des niveaux d'action et des niveaux d'exemption de l'intervention). Pour plus de commodité, ils peuvent être exprimés en termes de dose annuelle évitable, ou d'une grandeur connexe. Le recours à des niveaux de référence spécifiques prédéterminés peut permettre de prendre rapidement des décisions concernant les interventions et d'utiliser les ressources efficacement; les employer à mauvais escient pourrait cependant entraîner des entorses aux principes de justification et d'optimisation.

\subsection{Niveaux de référence génériques}

Le rapport recommande par ailleurs d'utiliser des niveaux de référence génériques pour les interventions. Par commodité, ces niveaux peuvent être exprimés en termes de dose annuelle existante. Ils sont particulièrement utiles lorsque l'on envisage une intervention dans certaines situations comme des expositions dues à un fond naturel de rayonnement élevé et à des déchets radioactifs hérités des temps anciens. Le rapport insiste cependant sur le fait que ces niveaux doivent être utilisés avec une extrême prudence. Si, dans la dose annuelle existante, il y a des composantes maîtrisables qui dominent clairement, le recours aux niveaux de référence génériques ne devrait pas empêcher que des actions protectrices soient engagées afin de réduire ces composantes dominantes. De telles actions peuvent être déclenchées par des niveaux de référence spécifiques ou des décisions au cas par cas, conformément aux prescriptions du système de protection radiologique concernant les interventions. Le recours aux niveaux de référence génériques ne devrait pas non plus inciter à utiliser les actions protectrices comme moyen de créer des « effets compensatoires » entre les différentes composantes. Une dose annuelle existante de faible niveau ne signifie pas nécessairement qu'il ne faut pas appliquer une action protectrice à l'une de ses composantes. Inversement, une dose annuelle existante de niveau élevé ne suppose pas nécessairement une intervention.

Avec ces réserves, les auteurs du rapport estiment qu'une dose annuelle existante d'environ $10 \mathrm{mSv}$ peut être utilisée comme niveau de référence générique au-dessous duquel une intervention ne sera probablement pas justifiable pour certaines situations d'exposition prolongée. Au-dessous de ce niveau, 


\section{Niveaux génériques d'intervention : dose annuelle existante}

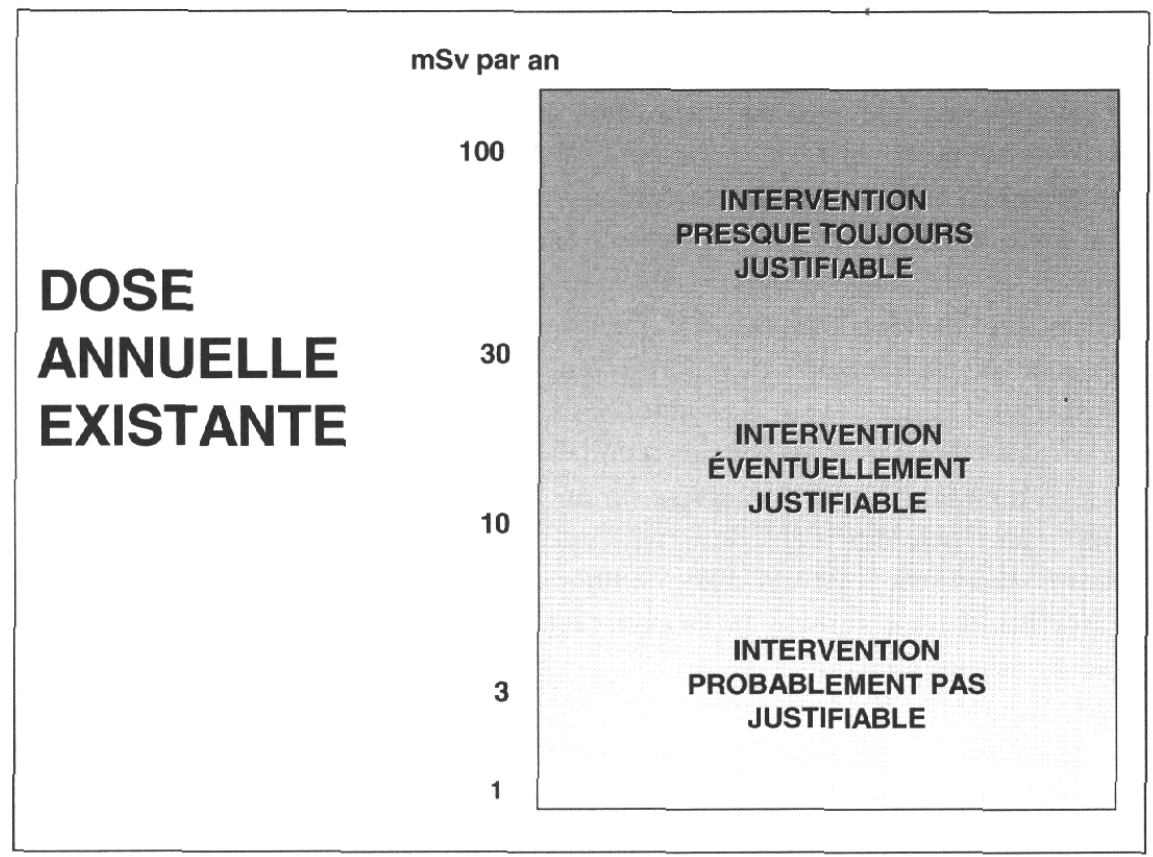

Figure 4 - Niveaux génériques d'intervention recommandés en termes de dose annuelle existant.

les actions protectrices visant à réduire une composante dominante de la dose annuelle existante restent facultatives et pourraient être justifiables. Dans de tels cas, on peut fixer des niveaux d'action propres aux différentes composantes sur la base des fractions correspondantes du niveau de référence générique recommandé. Au-delà du niveau au-dessous duquel une intervention n'est probablement pas justifiable, une intervention peut être nécessaire et elle devrait être justifiée au cas par cas. Dans les situations où les seuils annuels de dose (équivalente) s'appliquant aux effets déterministes sur les organes correspondants pourraient être dépassés, une intervention est nécessaire. Dans pratiquement toutes les circonstances imaginables, une dose annuelle existante se rapprochant de $100 \mathrm{mSv}$ justifiera presque toujours une intervention et cette valeur peut être utilisée comme niveau de référence générique pour engager des actions protectrices.

La figure 4 présente les niveaux d'intervention génériques recommandés. 


\section{Application à des situations particulières}

\subsection{Fond de rayonnement de niveau élevé}

Le rapport reconnaît que les situations d'exposition prolongée avec un fond naturel de rayonnement élevé et une exposition maîtrisable présentent un défi. On peut donner comme exemple un niveau élevé de radon 222 , isotope d'un gaz rare, à l'intérieur de bâtiments. Le rapport considère que les recommandations de la Commission sur la protection contre le radon 222 dans les habitations et sur les lieux de travail sont toujours valables et parfaitement applicables pour maîtriser l'exposition prolongée au radon 222 dans les habitations. Un autre exemple est la présence de radionucléides naturels émetteurs gamma dans les matériaux de construction et dans le sol. Les autorités nationales concernées et, le cas échéant, les organisations internationales compétentes devraient calculer des niveaux étalons d'exemption de l'intervention en fonction de l'activité massique de certains radionucléides dans les matériaux de construction, compte tenu des recommandations relatives aux produits contenant des substances radioactives présentées dans le rapport [voir ci-après]. Pour les zones où l'exposition à des émetteurs gamma naturels de niveau élevé dans le sol est maîtrisable, le rapport recommande d'utiliser à titre indicatif, pour résoudre des problèmes concrets, des fractions correspondantes des niveaux de référence génériques recommandés pour la dose annuelle existante.

\subsection{Déchets radioactifs}

D'autres situations difficiles d'exposition prolongée relevées dans le rapport sont dues à la présence de déchets radioactifs dans des habitations. Ces résidus peuvent résulter de l'arrêt ou du déclassement d'une pratique réglementée ou encore d'autres activités humaines ou événements passés, y compris les accidents. En ce qui concerne les pratiques réglementées, les contraintes et les limites de dose recommandées devraient être appliquées de manière prospective à l'exposition prolongée susceptible de subsister une fois la pratique arrêtée - au site d'une installation déclassée par exemple. (En principe, la contrainte de dose applicable ne devrait pas être supérieure à la contrainte de dose utilisée pendant la phase opérationnelle de la pratique. Toutefois, il n'y a pas nécessairement de base commune permettant de supposer une égalité entre la contrainte de dose appliquée avant et celle appliquée après l'arrêt de la pratique : si la contrainte de dose opérationnelle était très faible, son maintien après le déclassement pourrait entraîner une restriction déraisonnable.) En ce qui concerne les déchets radioactifs résultant d'autres activités humaines ou événements passés qui n'étaient pas réglementés en tant que pratiques, le besoin, le type, l'échelle et la durée des 


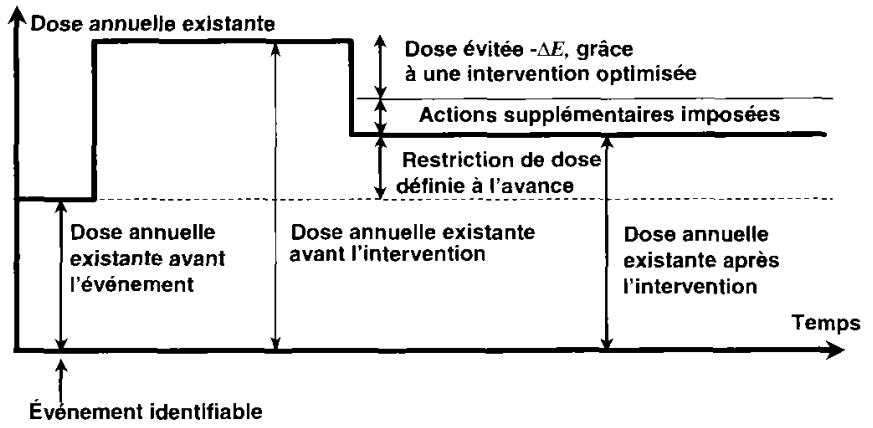

Figure 5 -Actions protectrices supplémentaires (s'ajoutant aux actions optimales) dans le cas d'événements identifiables.

actions protectrices devraient être déterminés au cas par cas. Pour cela, il conviendrait d'appliquer les principes recommandés de justification de l'intervention et d'optimisation des actions protectrices plutôt que des restrictions de dose individuelle définies à l'avance. Si nécessaire, on pourrait utiliser à titre indicatif les niveaux de référence génériques recommandés pour la dose annuelle existante. Toutefois, lorsqu'il est possible de remonter à l'origine de la situation et de faire endosser rétrospectivement la responsabilité des actions protectrices à ceux qui ont produit les déchets, les autorités nationales pourraient envisager d'appliquer une restriction spécifique aux doses individuelles imputables aux résidus afin de maintenir ces doses à des niveaux inférieurs à ceux qui résultent du processus d'optimisation. À cette fin, des actions protectrices supplémentaires peuvent être exigées de ceux qui sont à l'origine de la situation. Toutefois, cette restriction de dose spécifique peut être supérieure aux contraintes et limites de dose appliquées aux pratiques (Fig. 5). Les déchets dont on estime qu'ils ne nécessitent pas d'actions protectrices ne devraient pas faire l'objet de restrictions supplémentaires.

\subsection{Particules chaudes : expositions potentielles prolongées}

Dans certaines circonstances, la présence de déchets radioactifs dans l'environnement peut être très ponctuelle, généralement sous forme de «particules chaudes », ce qui donne lieu à des situations d'exposition potentielle prolongée. Ce sont des situations où existe la possibilité, mais non la certitude, d'une exposition. Le rapport recommande de calculer, dans de tels cas, les niveaux d'action en se fondant sur la probabilité inconditionnelle que les membres du public souffriraient d'effets sanitaires stochastiques mortels imputables à 


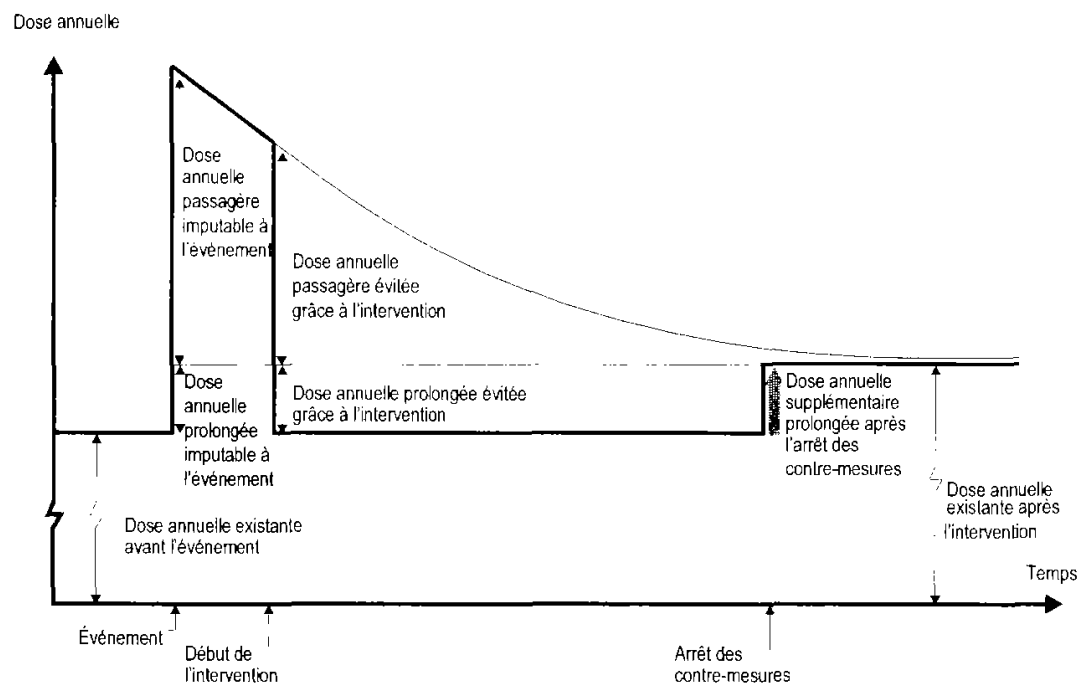

Figure 6 - Évolution de la dose annuelle dans le cas d'un accident suivi d'une intervention.

l'exposition. Cette probabilité devrait être calculée en combinant les probabilités suivantes: exposition à des particules chaudes; incorporation dans l'organisme d'une particule chaude à la suite de cette exposition; dose reçue à la suite de cette incorporation; effets stochastiques mortels consécutifs à cette dose. (Ces probabilités devraient être intégrées pour toute la gamme de situations et toute la gamme de doses possibles.) En fixant de tels niveaux d'action, il faudrait tenir compte de la possibilité que des effets déterministes localisés se produisent aussi à la suite de l'incorporation de particules chaudes.

\subsection{Accidents}

Des actions protectrices perturbatrices, telles que l'évacuation ou autres restrictions imposées aux conditions de vie «normales», peuvent s'avérer nécessaires après des accidents durant lesquels des substances radioactives ont été rejetées dans l'environnement (voir Fig. 6 pour l'évolution de la dose annuelle dans de tels cas). Tôt ou tard, pour retourner à la «normale », il est possible qu'il faille à un moment donné mettre fin à ces actions bien qu'une exposition prolongée résiduelle subsiste. Le moyen le plus simple de justifier l'arrêt d'une intervention après un accident est de confirmer que les expositions ont été ramenées aux niveaux d'action qui auraient déclenché l'intervention. Si une telle réduction de l'exposition n'est pas réalisable, le niveau de référence générique correspondant à la dose annuelle existante au-dessous de laquelle l'intervention n'est probablement 
pas justifiable, pourrait être la base sur laquelle fonder l'arrêt de l'intervention. Toutefois, il peut s'avérer difficile d'arrêter des actions protectrices qui sont en vigueur depuis de nombreuses années : la décision peut ne pas être acceptable pour la population exposée et les pressions sociales risquent de l'emporter sur l'avantage qu'il y aurait à arrêter l'intervention. Dans ce cas, la participation des parties prenantes au processus décisionnel devient indispensable. Après l'arrêt de l'intervention, la dose annuelle existante résiduelle ne devrait pas influer sur les conditions de vie normale dans la zone touchée (y-compris les décisions concernant l'introduction de nouvelles pratiques), même si cette dose est supérieure à celle qui prévalait dans la zone avant l'accident.

\subsection{Produits de consommation}

L'exposition prolongée peut être due à la présence de radionucléides de période longue dans les produits de consommation courante. Si les radionucléides sont liés à une pratique, leur niveau dans les produits devrait être maîtrisé en appliquant les principes du système de protection radiologique pour les pratiques. Dans les autres cas, les produits devraient théoriquement faire l'objet d'une intervention. En raison essentiellement de la mondialisation des marchés, les niveaux d'exemption de l'intervention pour les radionucléides dans les produits ne peuvent pas être fixés au cas par cas, mais doivent être normalisés. Il est peu probable que plusieurs types de produits soient des sources simultanées d'exposition prolongée élevée de quiconque. Partant de cette hypothèse, le rapport recommande un niveau générique d'exemption de l'intervention de l'ordre de $1 \mathrm{mSv}$ pour la dose annuelle individuelle résultant du type dominant de produit, comme certains matériaux de construction qui peuvent dans certaines circonstances être une cause non négligeable d'exposition prolongée. Sur la base de cette recommandation, les autorités nationales concernées et, le cas échéant, les organisations internationales compétentes, devraient calculer pour les différents produits, notamment pour certains matériaux de construction, des niveaux d'exemption de l'intervention spécifiques aux radionucléides. Il est indiqué dans le rapport que les niveaux d'exemption de l'intervention ne devraient pas servir, explicitement ou implicitement, à assouplir les limites concernant l'activité des radionucléides susceptibles d'être rejetés du fait de pratiques; en particulier, il ne faudrait pas avoir recours à ces niveaux pour autoriser le recyclage de matériaux provenant du déclassement de pratiques (les critères d'exemption pour les pratiques conviennent mieux dans ce cas).

La situation est exceptionnellement difficile dans le cas de produits fabriqués dans une zone touchée par des rejets radioactifs à la suite d'un accident, et contenant des substances radioactives imputables à ces rejets. Si les niveaux d'activité correspondants sont supérieurs à ceux des produits provenant de zones voisines, 
des questions d'acceptation de mise sur le marché peuvent se poser - notamment en cas de mouvement transfrontières des produits. (La Commission du Codex alimentarius OMS/FAO a adopté des niveaux génériques d'exemption de l'intervention pour les radionucléides présents dans les aliments à la suite d'un accident. Ces niveaux ont été pris en compte dans les normes internationales de protection radiologique. lls correspondent à des doses individuelles allant jusqu'à quelques millisieverts par an pour les consommateurs de tels aliments.) Si les doses annuelles dans la zone touchée par l'accident sont acceptables en raison de l'optimisation de la stratégie d'intervention, la situation en dehors de cette zone devrait être acceptable elle aussi, car les doses annuelles individuelles reçues en dehors de la zone touchée et imputables à l'utilisation de produits provenant de cette zone ne devraient pas être supérieures aux doses dans la zone touchée. Si les restrictions sur les produits provenant de la zone touchée par l'accident n'ont pas été levées, la production de ces derniers ne devrait pas redémarrer ; par contre, elle le peut si les restrictions ont été levées. Si un accroissement de la production est proposé, il doit être dûment justifié. Lorsque les restrictions ont été levées dans le cadre d'une décision de « retour à la normale », la reprise et l'accroissement éventuel de la production dans la zone touchée devraient avoir été examinés dans le cadre de cette décision et ne devraient plus avoir besoin de l'être.

\section{Résumé des recommandations quantitatives}

Les recommandations quantitatives formulées dans le rapport sont résumées dans le tableau I. Les informations sont présentées de manière très simplifiée et ne se prêtent pas à des comparaisons. Dans la partie supérieure du tableau, les recommandations quantitatives sont exprimées en termes de dose annuelle existante individuelle; dans la partie inférieure, elles le sont en termes de dose annuelle supplémentaire individuelle. De ce fait, les fourchettes de doses figurant dans les deux parties du tableau sont exprimées dans des grandeurs différentes et ne peuvent pas être comparées. En outre, le tableau ne fait aucune référence à des niveaux d'intervention ou d'action spécifiques correspondant à la dose annuelle évitée ou à des doses collectives.

\section{Mise en ouvre des recommandations}

Le rapport reconnaît que les recommandations quantitatives seront difficiles à mettre en œuvre si un certain nombre de questions relatives à l'estimation des expositions ne sont pas soigneusement prises en compte. En général, les expositions prolongées sont évaluées sur la base de la dose annuelle moyenne dans le groupe critique. Toutefois, il convient de noter qu'il peut être parfois plus difficile d'évaluer cette dose que la dose reçue par une personne ayant subi une exposition «maximale». Des scénarios à long terme doivent être définis pour caractériser les personnes exposées et le type de l'exposition qu'elles ont subie. 
TABLEAU I

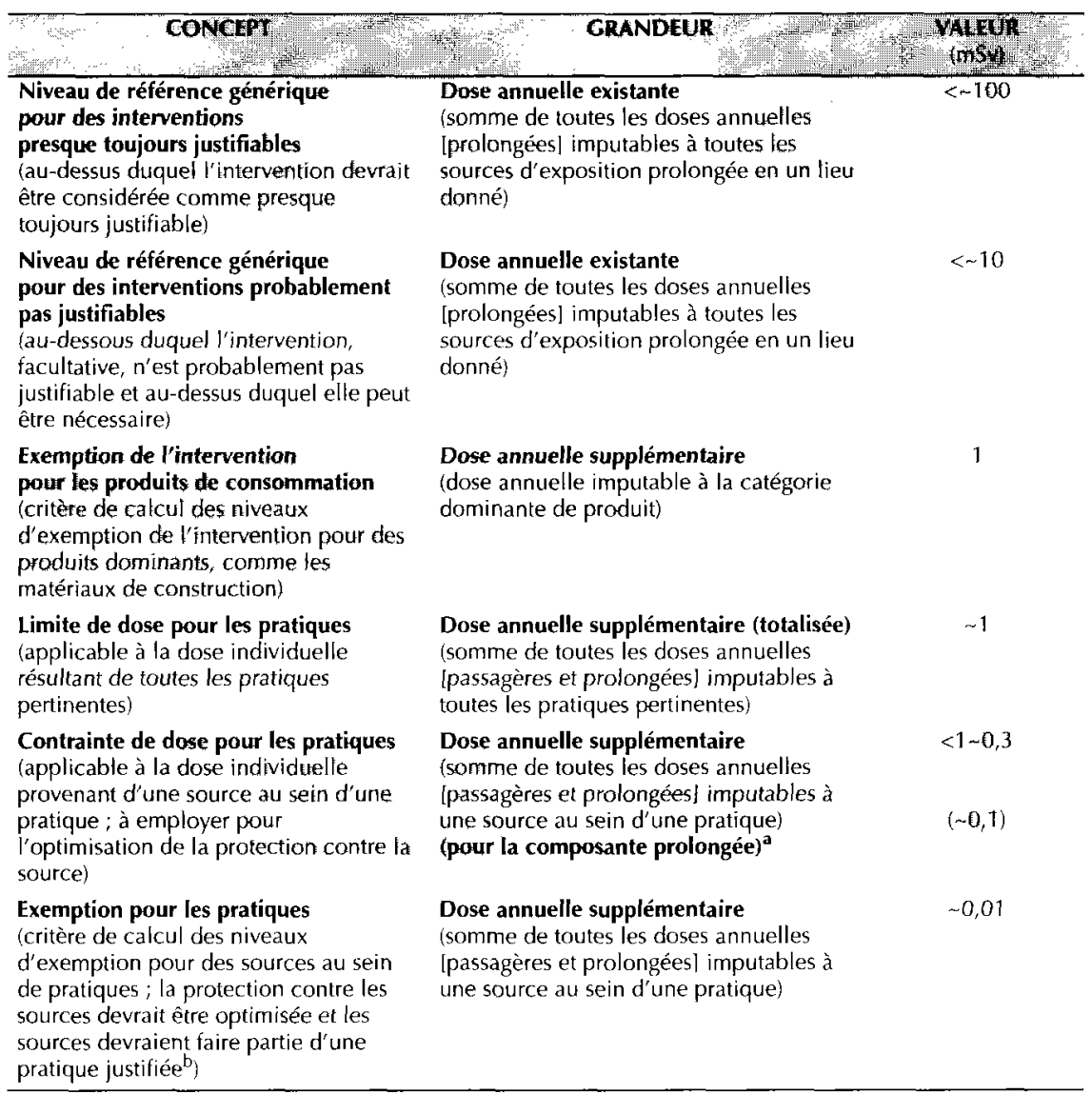

\footnotetext{
¿ À envisager en l'absence de méthodologies d'évaluation des doses pour assurer la conformité quelle que soit la combinaison de doses.

b Applicable aussi généralement pour autoriser le rejet de matériaux contenant des substances radioactives résultant de pratiques et susceptibles ultérieurement d'être recyclées comme produits de consommation sans restrictions.
}

La quantification de l'incertitude devrait faire partie intégrante de la détermination des doses annuelles. Chaque fois que cela est possible et approprié, il faudrait indiquer les doses annuelles en tant que distribution de valeurs possibles plutôt qu'en tant que valeurs ponctuelles. Il ne faut pas oublier que la répartition des déchets radioactifs est généralement inégale, ce qui engendre des situations d'exposition prolongée hétérogène. Celles-ci doivent être abordées au cas par cas en formulant des hypothèses réalistes quant au schéma d'exposition des personnes. Le choix des méthodes à retenir pour évaluer l'exposition hétérogène dépendra de 
la situation et des objectifs de l'évaluation. L'évaluation des doses annuelles dans des situations d'exposition prolongée devrait en général reposer sur l'hypothèse d'un usage non restreint du site ou des produits touchés; cette hypothèse suppose la prise en compte de toutes les voies d'exposition qui pourraient être effectives un jour.

\section{Perspectives}

Les recommandations formulées dans le rapport de la CIPR reposent sur des évaluations objectives des risques sanitaires liés à des niveaux d'exposition prolongée et tiennent compte de dimensions de la protection radiologique liées à diverses situations d'exposition. Toutefois, les membres du public (et parfois leurs représentants politiques) peuvent avoir des points de vue personnels divergents sur les risques radiologiques imputables à des sources artificielles d'exposition prolongée comparés aux risques imputables à des sources naturelles. Cela se traduit habituellement par une perception différente de la réponse requise et par une échelle de protection différente, suivant l'origine de l'exposition. La demande de protection est généralement plus ferme lorsque la source de l'exposition est un sous-produit technologique que lorsqu' on considère qu'elle est d'origine naturelle. Ainsi, les expositions prolongées élevées qui sont imputables à des sources naturelles de rayonnements sont généralement ignorées par la société, alors que des expositions prolongées, relativement faibles, dues à des déchets radioactifs de période longue constituent une source de préoccupation et donnent lieu parfois à des actions superflues. Ces dimensions sociales et politiques, le plus souvent sans rapport avec la protection radiologique, influent généralement sur la décision définitive quant au niveau de protection approprié dans le cas d'une exposition prolongée. C'est pourquoi le rapport de la CIPR reconnait prudemment qu'il devrait être considéré comme contenant des recommandations d'aide à la décision, fondées essentiellement sur des considérations scientifiques de protection radiologique, les résultats devant en principe servir au processus final de prise de décisions (généralement plus large), qui peut faire intervenir d'autres questions et considérations intéressant la société. Le processus de prise de décision pour la protection radiologique du public dans des situations d'exposition prolongée peut faire appel aux diverses parties prenantes et pas seulement aux radioprotectionnistes.

\section{RÉFÉRENCE}

ICRP Publication 82 (1999) Protection of the public in situations of prolonged radiation exposure: the application of the commission's system of radiological protection to controllable radiation exposure due to natural sources and long lived radioactive residues (Protection du public dans des situations d'exposition prolongée à des rayonnements : application du système de protection radiologique de la Commission aux expositions maîtrisables dues à des sources naturelles et à des résidus radioactifs de période longue), Ann. ICRP 82 (1-2). 